\title{
Pharmacognostical evaluation of indigenous herbal drug: Carissa carandas Linn. fruit
}

\author{
Sonia Singh ${ }^{\star}$, Meenakshi Bajpai and Pradeep Mishra \\ Institute of Pharmaceutical Research, GLA University, NH-2, Mathura-Delhi Road, Chaumuhan-281406, Mathura, Uttar Pradesh, India
}

\section{Article Info}

\section{Article history}

Received 11 September 2020

Revised 28 October 2020

Accepted 30 October 2020

Published online 30 December 2020

\section{Keywords}

Carissa carandas L.

Organoleptic

Microscopy

Physicochemical

Ash value

Fluorescence analysis

\begin{abstract}
Carissa carandas Linn. (Family: Apocynaceae), commonly called Karonda in the northern region of India. In the present work, the organoleptic, anatomy profiles and antiradical activity of $C$. carandas fruits, as these parameters are concerned with benefit approaches in Indian medicinal plants for the standardization of crude drugs. It is widely used for edible purposes in the northern regions of India in the form of pickles and curry. The essential parameters used up for the pharmacognosy of given fruit were macroscopical study, histochemical evaluation, including transverse section, powder analysis, preliminary phytochemical and fluorescence analysis. The total phenolic and total flavonoid contents in $C$. carandas fruit were determined using Folin-Ciocalteu method and Aluminium chloride colorimetric assay. The organoleptic and macroscopic profiles of fruit revealed the presence of petiole, the smooth outer surface, green color with a sour taste. The histochemical evaluation showed the presence of pericarp and endosperm. Sclereids, oil globules, calcium oxalate crystals and sclerenchymatous cells found in the fruit section. Alkaloids, saponin glycosides, flavonoids, tannins, and phenolic compounds, fats, and oils, volatile oils, carbohydrates, gums, mucilage, and reducing sugars were present. The physicochemical evaluation such as moisture content, ash value, extractive value, foreign matter, and fluorescence analysis were performed and recorded. The fruit extracts of plant showed the presence of phenolic and flavonoid content. Data obtained used as a standard for future aspects. The current report on pharmacognostic, preliminary physicochemical analysis provides an important diagnostic tool and evidence in correct identification, and even standardization of crude drug.
\end{abstract}

\section{Introduction}

India is well known for it to be the house for traditional herbal medicines. Their presence well documented in Ayurveda, Siddha, and Unani. About $60 \%$ of the world's population is using the alternative system of medicines. The rural community eventually uses such medications for primary healthcare issues but also used by developed and developing countries wherever modern medicines do exist. Looking at the immense applications with less possible side effects, the whole world bent upon using herbals for medicinal use (Pandey et al., 2013).

World Health Organization has identified and listed 21,000 plants, which used for medicinal applications. Approximately 2500 species are of their indigenous to India, out of which 150 species have already been utilized commercially on a large scale. India is well known as the botanical garden of the world and considered as the largest exporter and importer of herbal drugs (Modak et al., 2007).

Corresponding author: Ms. Sonia Singh Assistant Professor, Department of Pharmacognosy, Institute of Pharmaceutical Research, GLA University, NH-2, Mathura-Delhi Road, Chaumuhan-281406, Mathura, Uttar Pradesh India

E-mail: sonia.singh@gla.ac.in

Tel.: +91-9997066034

Copyright (C) 2020 Ukaaz Publications. All rights reserved.

Email: ukaaz@yahoo.com; Website: www.ukaazpublications.com
The plant of this article, C. carandas (Family: Apocynaceae) is an annual shrub containing small spikes. It is native to Pakistan, India, Bangladesh, Sri Lanka, Java, Malaysia, Myanmar, and Nepal (Tesfaye and Ravi Chandran, 2018).

The fruits are commonly used as vegetable in India. The extracts are mainly used in the treatment of hepatic diseases, used to reduce the increased body temperature. Additionally, the roots and fruits are employed as digestant, astringent, vermifuge, insect repellant, stomachic, appetizer, diuretic (Saha et al., 2011) anti-inflammatory and aphrodisiac; as an antiscorbutic agent (Singh and Uppal, 2015; Agarwal et al., 2012; Hati et al., 2014).

The crude drug possessed to contain $\beta$-sitosterol, carindone, steroids, terpenes, lupeol, cardenolides, carissone, flavonoids, tannins, benzenoids, lignans, phenylpropanoid, coumarins and sesquiterpenes (Arif et al., 2016; Anupama et al., 2014; Begum et al., 2013; Sadek et al., 2013; Virmani et al., 2017).

From ancient time period, the plants have been employed as a wonderful source of many natural herbal products for the treatment and prevention of human health (Kumar et al., 2005). World Health Organization (WHO) (Atanassova et al., 2011) has suggested that such exercise of using natural herbs should be encouraged in most of the places where the access of conventional therapy and treatment is not adequate and enough. Researchers found that several plants 
have secondary metabolites and therapeutic activities which produce physiological actions in the human body, thereafter they could be utilized in the treatment of various ailments (Omale and Okafor, 2008). Such bioactive chemical constituents are alkaloids, glycosides, tannins, flavonoids and phenolic compounds (Atanassova et al., 2011; Edeoga et al., 2005). Flavonoids, one of the bioactive components containing free radical properties, inhibiting various hydrolytic and oxidative enzyme activities (Atanassova et al., 2011). Because of the presence of such polyphenolic components, many plants have shown potent antioxidant activities (Cook and Samman, 1996). Medicinal plants containing antioxidant properties help in scavenging the free radical generated via oxidative stress and also involved in the protection of degenerative disorders (Mimica-Dukic et al., 2004).

Though, Chanchal et al. ( 2013) has discussed the pharmaconostic study of $C$. carandas fruits and roots, but the work has not completely provided detailed information about the microscopy sectional study of fruit with different reagents. The present experimental work deals with detail pharmacognostical studies of the plant; comparative evaluation of total phenolic and total flavonoid content between ethanolic and aqueous fruit extract of C. carandas. This would help in the authentication of crude drug that might be used in the herbal formulations.

\section{Materials and Methods}

\subsection{Collection and authentication of plant material}

Fresh fruits of Carissa carandas Linn. were procured from local markets of Mathura, Uttar Pradesh (India) in the month of June. The fruits were certified by NISCAIR, New Delhi and deposited at the herbarium of raw materials herbarium and museum, Delhi (RHMD) as voucher specimen with Ref. No. NISCAIR/RHMD/ Consult/2015/2925-1183-3 for future analysis.

\subsection{Chemicals used for analysis}

Toluidine blue O reagent, chloral hydrate reagent, phloroglucinol reagent, N/50 iodine solution, glycerine, concentrated hydrochloric acid, sulphuric acid, ethanol, chloroform, benzene, petroleum ether $\left(60-80^{\circ}\right)$, and all other analytical grades of chemicals were purchased and used for the study from Sisco Research Laboratories Pvt. Ltd. and Sigma-Aldrich, India.

\subsection{Macroscopic evaluation}

Macroscopic analysis of fruits such as form, shape, color, odor, taste, and other parameters were observed and studied as per the method of Iyengar and Nayak (1997) and Khandelwal et al. (2012).

\subsection{Microscopic evaluation}

\subsubsection{Transverse section of $C$. carandas fruits}

With the help of microtome, the transverse section of fruit obtained and stained with different reagents to distinguish various characters. The stained slides were analyzed under a phase-contrast microscope (under total magnification power of 100X and 450X).

\subsubsection{Study of histochemical powder characteristic}

Slides of dried fruit powder were prepared in different reagents and visualized under phase contrast microscope for powder analysis.

\subsection{Physicochemical evaluation}

The physicochemical parameters such as ash value, acid ash value, swelling index, moisture content, extractive value, and foreign matter analyzed according to the methods described in WHO (1992).

\subsection{Preliminary phytochemical evaluation}

The preliminary phytochemical study of ethanolic and aqueous extracts of the dried powder performed for the presence of different chemical components as per the standard procedure mentioned by Kokate (2005).

\subsection{Preparation of herbal extracts using different solvents}

Fresh fruits were washed thoroughly to remove any adherent impurities. The fruits were then cut into small pieces and allowed to dry in the shade. Dried fruits were then powdered using an ordinary grinder. The powdered material sieved through $\# 40$ mesh size. It was defatted using petroleum ether $\left(60-80^{\circ}\right)$ in Soxhlet apparatus. The defatted powder is then undergone extraction process using different solvents such as benzene, chloroform, ethyl acetate, and methanol. Later on, the remaining residue was macerated with chloroform water to obtain the aqueous extract. The extracts obtained were concentrated and finally evaporated to dryness on a water bath. The percentage yield was calculated (in terms of the air-dried weight of the plant material) for each obtained extracts. The consistency and color of the extracts were also recorded (Chatterjee and Satyesh, 1994; Rangari, 2000).

\subsection{Fluorescence evaluation}

The fluorescence analysis of dried fruit powder and fruit extracts were treated with different chemical reagents such as $50 \%$ sulphuric acid, 50\% nitric acid , 5\% ferric chloride, concentrated hydrochloric acid, acetic acid, 5\% sodium hydroxide were examined under daylight, short-wavelength, and long-wavelength to analyze the fluorescence behavior of the fruit (Evan, 2006; Kokate, 1999).

\subsection{Determination of total phenolic content}

Total phenolic content (TPC) was evaluated for each extracts using Folin-ciocalteu method. $1 \mathrm{ml}$ of each extract $(10-100 \mu \mathrm{g} / \mathrm{ml})$ solution was admixed with $3 \mathrm{ml}$ of Folin-Ciocalteu reagent in the volumetric flask. $3.5 \mathrm{ml}$ sodium carbonate $(7.5 \% \mathrm{w} / \mathrm{v})$ was added to the mixture and then it has been incubated at room temperature for $30 \mathrm{~min}$ with constant agitation. The absorbance of the resultant mixture was measured at $765 \mathrm{~nm}$ under UV spectrophotometer (Elizabeth and Kelly, 2007). The data obtained for TPC of C. carandas fruits were expressed as milligrams of gallic acid (GAE)/g fruit extract wt. Samples were analysed in triplicates.

\subsection{Determination of total flavonoid content}

The flavonoid contents (TFC) of extracts were determined as per method outlined in Ling-jia et al. (2018) $1 \mathrm{ml}$ of test extracts or rutin $(10-100 \mu \mathrm{g} / \mathrm{ml})$ were allowed to mix with $0.2 \mathrm{ml}$ of $10 \% \mathrm{w} / \mathrm{v}$ $\mathrm{AlCl}_{3}$ in methanol, $0.5 \mathrm{ml}$ sodium hydroxide solution and $6 \mathrm{ml}$ of distilled water in the volumetric flask. The mixtures were incubated for $30 \mathrm{~min}$ at room temperature. The absorbance was determined under UV spectrophotometer at $415 \mathrm{~nm}$ (Ling-jia et al., 2018). The data obtained for TFC of C. carandas fruits were expressed as milligrams of rutin/g fruit extract wt. Samples were analysed in triplicates. 


\subsection{Statistical analysis}

The experimental work was carried out in triplicates and the results were calculated and expressed as mean \pm SD (standard deviation).

\section{Results}

\subsection{Macroscopic characters}

The fruits are reddish, pinkish-white and of globular shape. The shape comprises of at times oval to the elliptical shape. The number of seeds was found to be 2 to 4 per fruit. The size of the fruit is approximately 2.0 to $2.5 \mathrm{~cm}$ (Figure 1). It has a sour and acrid taste. The seeds are blackish-brown in color with an elliptical shape. The plant starts flowering in the mid of February and bearing fruits. These get matured in July to August. The fruits are generally harvested at their immature stage for edible usage in pickles, curry, and chutneys rather than ripened one.

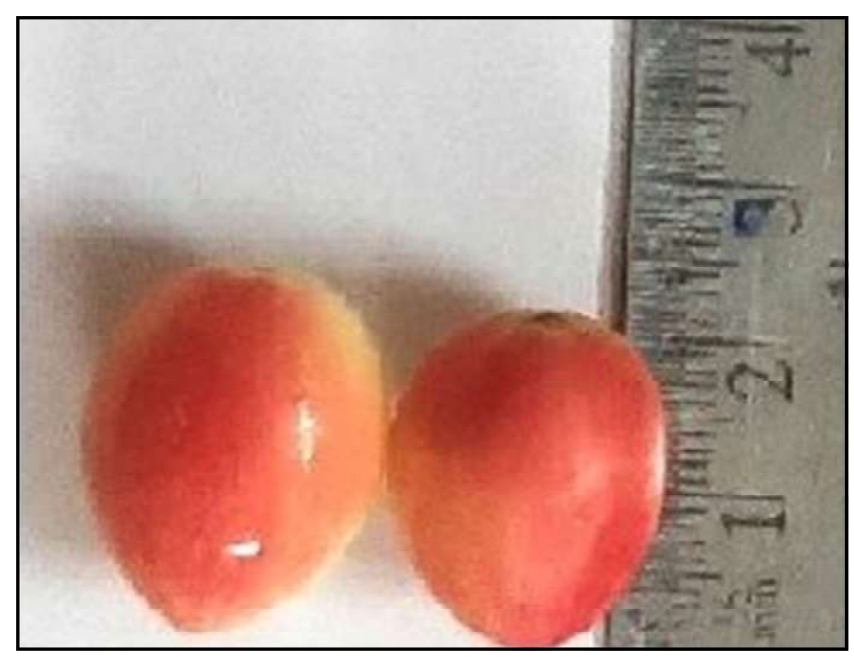

Figure 1: Macroscopy of C. carandas Linn fruits.

\subsection{Microscopic characters}

Transverse section of fruit shows a well-differentiated zone, namely; pericarp and endosperm (Figure 2). Pericarp consists of an external epicarp, a broad region of parenchymatous mesocarp and a single layer of endocarp, respectively.

Epicarp has few layers of thick-walled of epidermal cells, which are followed by a thick layer of cuticle. It has an outer, densely arranged regions, called flavido and interior consists of loosely organized cells.

Mesocarp surface appeared as a big band of tangentially elongated parenchymatous cells in which many isolated oil globules visualized. These oil globules are scattered allover the section, but few of them seen in the inner region of mesocarp. Flavidovascular bundles visualized in the inner part of mesocarp (Figure 3). These bundles are found lignified when treated with phloroglucinol and concentrated hydrochloric acid (1:1). The section also contains prism of calcium oxalate crystals. The lysigenous cavities broadly distributed in mesocarp region, as some of the pits also found in the epicarp region.
Endocarp forms the bulk of the seed with thick-walled polygonal parenchymatous cells containing aleurone grains and fixed oil (Figures 4 and 5).

Section when stained with toluidine blue O reagent, it showed the presence of large cells with plasmodesmata pit region as seen in Figure 5. Even the section had retained the blue color dye which showed precise characterization of cuticle, epidermis, mesocarp with large cells and sclereids (Figure 4).

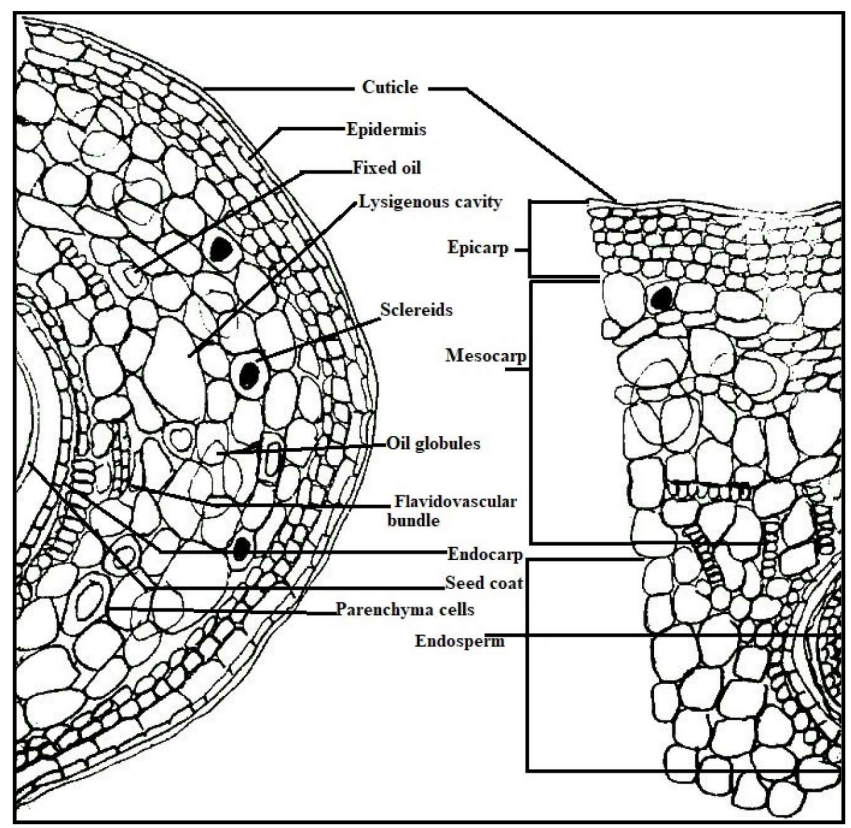

Figure 2: Transverse section of $C$. carandas Linn. fruit.

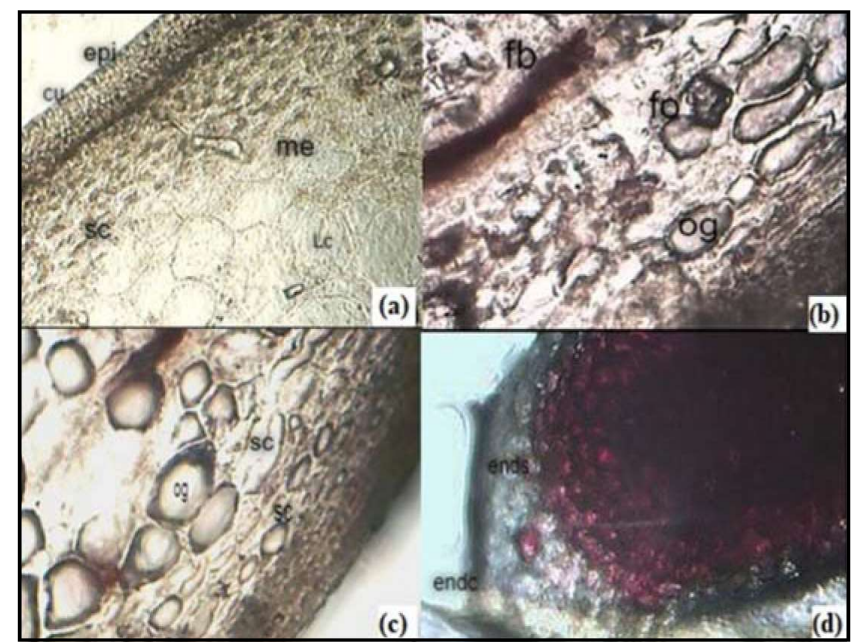

Figure 3: Transverse section of C. carandas Linn. fruit at 100X, stained with phloroglucinol reagent and concentrated hydrochloric acid (1:1). a) cuticle (cu) with epidermal cells (epi), presence of mesocarp (me), lysigenous cavity (Lc); b) and c) show the presence of oil globules (og), fixed oil (fo) followed by flavidovascular bundles (fb) and sclereids (sc); d) endocarp (endoc) with endosperm (ends). 


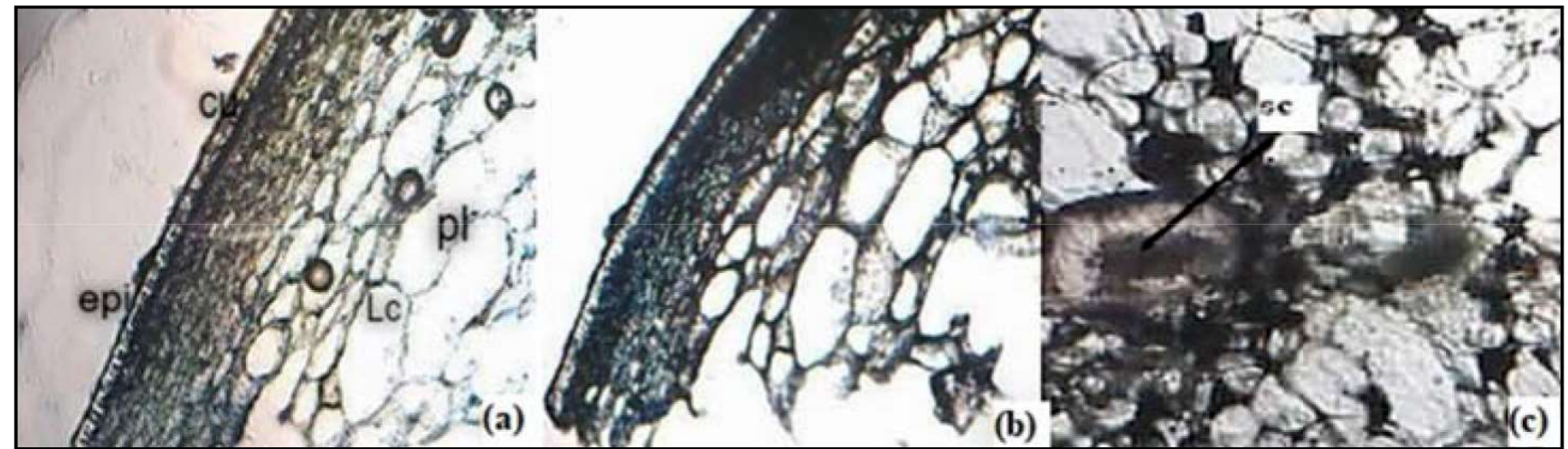

Figure 4: Transverse section of C. carandas Linn. fruit at 100X, stained with toluidine blue O reagent. (a) and (b) epidermis (epi) with thin cuticle (cu), presence of large cells (Lc) with plasmodesmata pit (pl); (c) sclereids (sc).

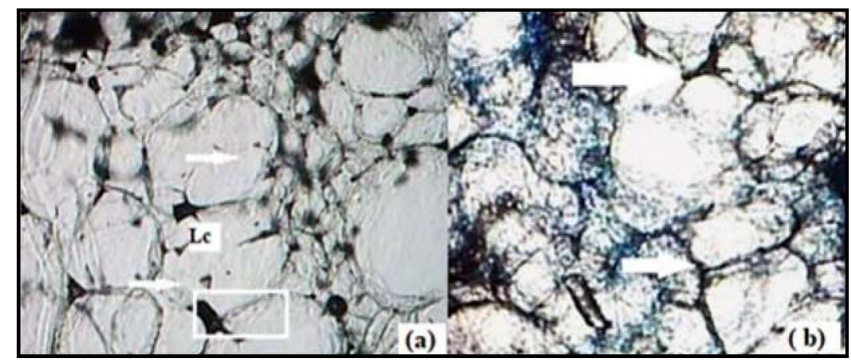

Figure 5: Sections of the pericarp of $C$. carandas Linn. fruit stained with toluidine blue $\mathrm{O}$ reagent (a) show retention of staining in cell walls bounded with large cells (Lc) and observed plasmodesmata pit $(\mathrm{pl})$ regions (arrows).

\subsection{Powdered drug characters}

The powder fruit material is brownish with reddish tinge appearance. It shows the fragments of parenchyma cells, epidermal cells, lignified flavidovascular bundles, mesocarp cells along with oil globules (Figure 6).

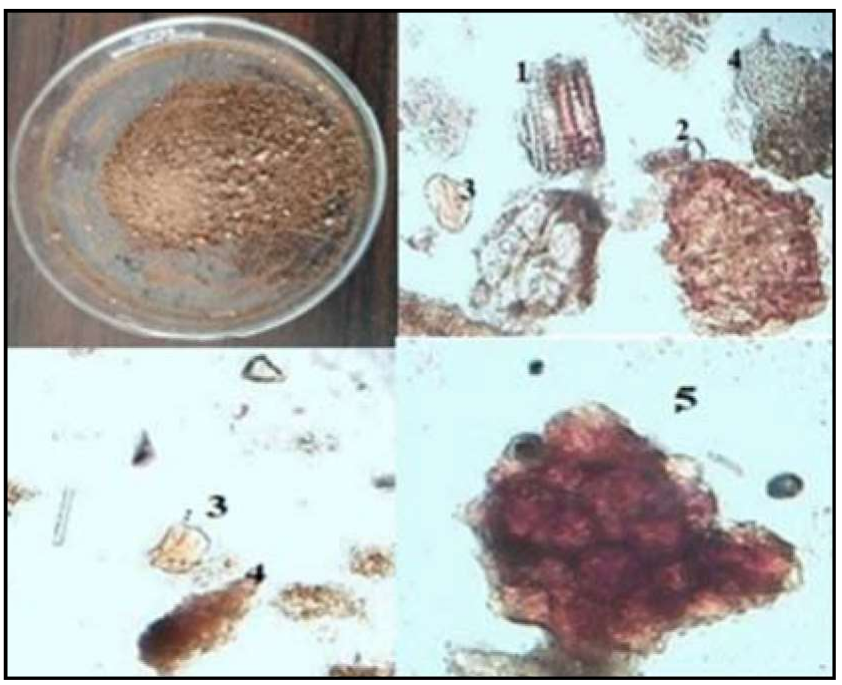

Figure 6: Powder analysis of C. carnadas Linn. fruit: 1. Flavidovascular bundle; 2. epidermal cell; 3. Oil globules; 4. Mesocarp cells; 5. Parenchyma cell.

\subsection{Physicochemical evaluation}

The values obtained during the physicochemical analysis are recorded and mentioned in Table 1, Figures 7 and 8 . The fruit powder contains $9.69 \%$ of moisture content, $4.0 \mathrm{ml}$ of the swelling index, $6.42 \% \mathrm{w} / \mathrm{w}$ of total ash value, $0.45 \% \mathrm{w} / \mathrm{w}$ of acid-insoluble ash value, $1.37 \% \mathrm{w} / \mathrm{w}$ of water-insoluble ash value and $0.07 \%$ of foreign matter, respectively. The further investigation revealed that the extractive values for different solvents such as petroleum ether, benzene, chloroform, ethanol, and aqueous extracts were found to be $15.026 \%, 1.746 \%, 0.776 \%, 9.304 \%$, and $22.4 \%$.

Table 1: Physicochemical evaluation of Carissa carandas Linn. fruits

\begin{tabular}{|c|l|c|}
\hline SI.No. & Physicochemical parameters & Results \\
\hline 1. & Loss on drying $(\% \mathrm{w} / \mathrm{w})$ & $9.69 \pm 0.23$ \\
\hline 2. & Swelling index $(\mathrm{ml})$ & $4.00 \pm 0.06$ \\
\hline 3. & Total ash value $(\% \mathrm{w} / \mathrm{w})$ & $6.42 \pm 0.38$ \\
\hline 4. & Acid-insoluble ash value $(\% \mathrm{w} / \mathrm{w})$ & $0.45 \pm 0.02$ \\
\hline 5. & Water-insoluble ash value $(\% \mathrm{w} / \mathrm{w})$ & $1.37 \pm 0.34$ \\
\hline 6. & Foreign organic matter $(\% \mathrm{w} / \mathrm{w})$ & 0.07 \\
\hline 7. & Extractive value $(\%$ yield $)$ & 15.026 \\
\hline (a) & Petroleum ether extract $\left(40-60^{\circ} \mathrm{C}\right)$ & 1.746 \\
\hline (b) & Benzene extract & 0.776 \\
\hline (c) & Chloroform extract & 9.304 \\
\hline (d) & Ethanol extract & 22.4 \\
\hline (e) & Aqueous extract \\
\hline
\end{tabular}

$\mathrm{n}=03$; Values are expressed as Mean \pm SD.

\subsection{Preliminary phytochemical evaluation}

The screening of phytochemical studies revealed the presence of alkaloids, saponin glycosides, flavonoids, tannins, and phenolic compounds, fats, and oils, volatile oils, carbohydrates, gums, and mucilage, reducing sugars (Table 2).

\subsection{Fluorescence analysis}

The fluorescence evaluation of $C$. carandas powdered fruit and different extracts recorded in Table 3. This parameter provides some valuable information about the purity of crude drugs while observing under visible light and UV light, respectively. 


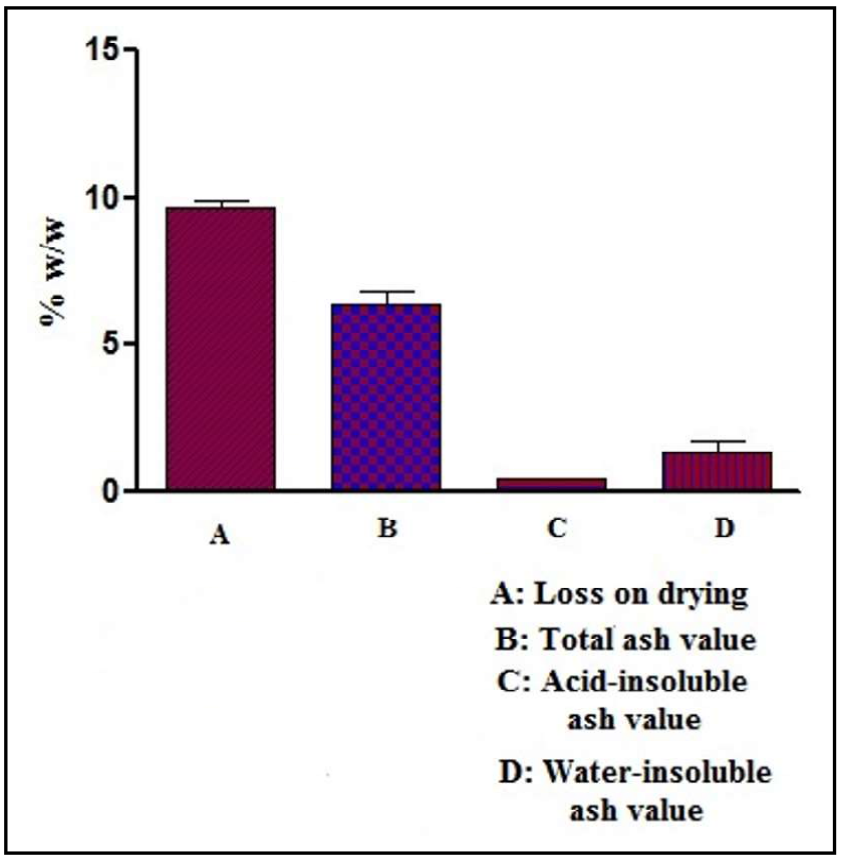

Figure 7: Physicochemical parameters of C. carandas fruit.

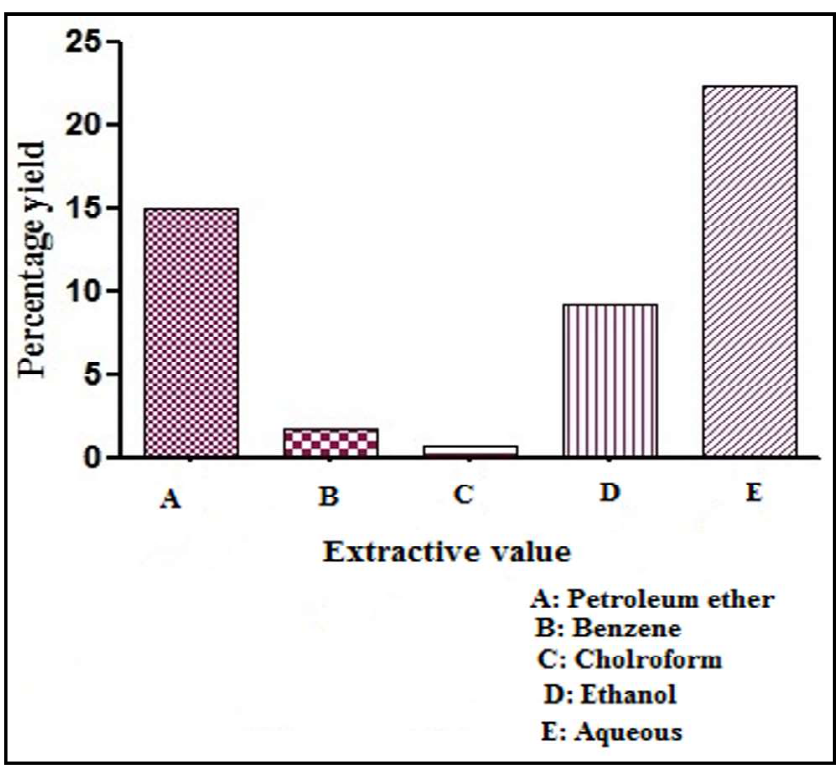

Figure 8: Extractive values of C. carandas fruit.

\subsection{Total phenolic and total flavonoid content}

Ethanolic and aqueous extract of $C$. carandas fruits were analyzed for their total phenolic and total flavonoid content as shown in Table 4. Quantitative estimation of the polyphenolic components of C. carandas fruit extract showed that the fruits are rich in phenolic and flavonoid content. The amounts of total phenolic and total flavonoid in Karonda (C. carnadas) were found to be 228.23 to $324.96 \mathrm{mg} \mathrm{GAE} / 100 \mathrm{~g}$ and 352.06 to $428.25 \mathrm{mg}$ Rutin/ $100 \mathrm{~g}$ respectively, as mentioned in Table 4 with gallic acid and rutin (Figure 9 and Figure 10) as standards.
Table 2: Phytochemical screening of different extracts of C. carandas fruits.

\begin{tabular}{|c|c|c|c|}
\hline \multirow[t]{2}{*}{ SI.No. } & \multirow[t]{2}{*}{ Chemical tests } & \multicolumn{2}{|c|}{ Extracts } \\
\hline & & Ethanol & Aqueous \\
\hline \multicolumn{4}{|c|}{ Cardiacglycosides } \\
\hline (a) & Keller Killani test & - & - \\
\hline (b) & Legal's test & - & - \\
\hline \multicolumn{4}{|c|}{ Anthraquinoneglycosides } \\
\hline (a) & Borntrager's test & - & - \\
\hline (b) & Modified Borntrager's test & - & - \\
\hline \multicolumn{4}{|c|}{ Saponinglycoside } \\
\hline (a) & Foam test & ++ & + \\
\hline \multicolumn{4}{|c|}{ Flavonoids } \\
\hline (a) & Shinoda test & +++ & ++ \\
\hline (b) & Lead acetate test & + & + \\
\hline \multicolumn{4}{|c|}{ Tannins and phenolic compounds } \\
\hline (a) & Ferric chloride solution & +++ & ++ \\
\hline (b) & Gelatin solution & ++ & ++ \\
\hline (c) & Lead acetate solution & + & - \\
\hline (d) & dilute nitric acid & +++ & ++ \\
\hline \multicolumn{4}{|c|}{ Protein and amino acids } \\
\hline (a) & Biuret test & - & - \\
\hline (b) & Ninhydrin test & - & - \\
\hline \multicolumn{4}{|c|}{ Fats and Oils } \\
\hline (a) & Solubility test & + & - \\
\hline (b) & Filter paper stain test & + & - \\
\hline \multicolumn{4}{|c|}{ Volatile oils } \\
\hline (a) & Filter paper stain test & + & - \\
\hline \multicolumn{4}{|c|}{ Carbohydrates } \\
\hline (a) & Molisch's reagent & ++ & ++ \\
\hline \multicolumn{4}{|c|}{ Gums and mucilages } \\
\hline (a) & Molisch's test & + & + \\
\hline \multicolumn{4}{|c|}{ Reducing sugars } \\
\hline (a) & Fehling's test & + & + \\
\hline (b) & Benedict's test & + & + \\
\hline \multicolumn{4}{|c|}{ Monosaccharides } \\
\hline (a) & Barfoed's test & - & - \\
\hline \multicolumn{4}{|c|}{ Alkaloids } \\
\hline (a) & Mayer's reagent & ++ & + \\
\hline (b) & Dragendorff's reagent & + & ++ \\
\hline (c) & Hager's reagent & ++ & + \\
\hline (d) & Wagner's reagent & ++ & + \\
\hline
\end{tabular}

Chemical identification of C.carandas extracts: '+'? positive test; '-': negative test. 
Table 3: Fluorescence analysis of $C$. carandas fruit (powder and extracts)

\begin{tabular}{|c|l|l|l|l|}
\hline \multirow{2}{*}{ SI.No. } & Treatment & \multirow{2}{*}{ Visible light } & \multicolumn{2}{|c|}{ UV light } \\
\cline { 3 - 5 } & & & Short 254 nm & Long 365 $\mathbf{~ n m}$ \\
\hline 1 & Powder + 5\% Sodium hydroxide & Brown & Dark brown & White \\
3 & Powder $+50 \%$ Sulphuric acid & Pink & Light brown & White \\
4 & Powder $+50 \%$ Nitric acid & Pight brown & White & Green \\
5 & Powder + 5\% Ferric chloride & Brown & Light yellow & Dark yellow \\
6 & Powder + Acetic acid & Pink & Light brown & Dark brown \\
7 & Petroleum ether extract $\left(40-60^{\circ} \mathrm{C}\right)$ & Dark brown & Yellow & White \\
8 & Benzene extract & Green & Light green & Dark brown \\
9 & Chloroform extract & Green & Yellow & White \\
10 & Ethanol extract & Gight brown & Green & White \\
11 & Aqueous extract & Dark brown & White & White \\
12 & Drug powder & Brown & Brown & Off white \\
\hline
\end{tabular}

Table 4: Total phenolic and flavonoid contents of C. carandas fruit extracts

\begin{tabular}{|l|l|l|l|}
\hline Sl.No. & Extract & TPC (mg GAE/g fruit extract wt) & TFC (mg Rutin/g fruit extract wt) \\
\hline 1 & Ethanolic fruit extract C. carandas & $324.96 \pm 0.02$ & $428.25 \pm 0.45$ \\
2 & Aqueous fruit extract C. carandas & $228.23 \pm 0.34$ & $352.06 \pm 0.18$ \\
\hline
\end{tabular}

$\mathrm{n}=3$; TPC: total phenolic content; TFC: total flavonoid content; GAE: gallic acid equivalent; wt: weight.

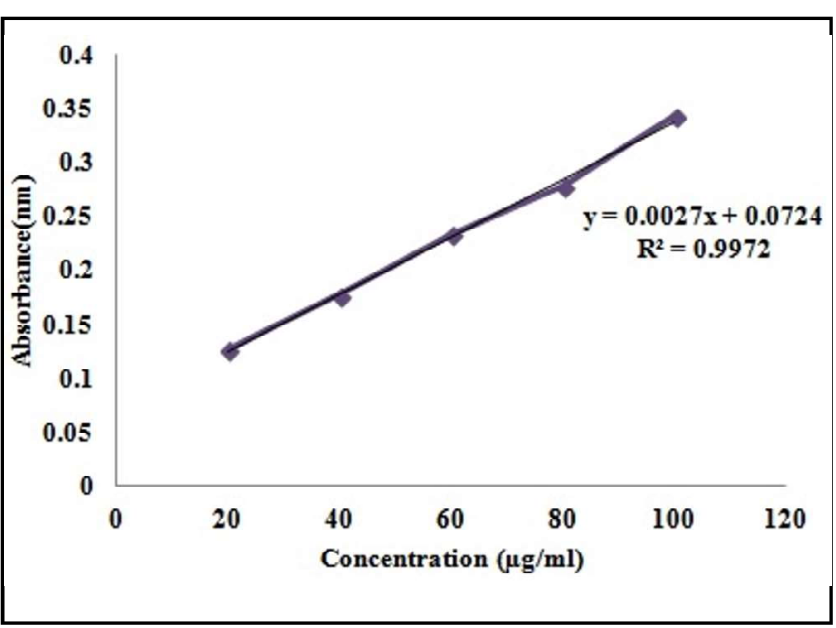

Figure 9: Caliberation of Gallic acid.

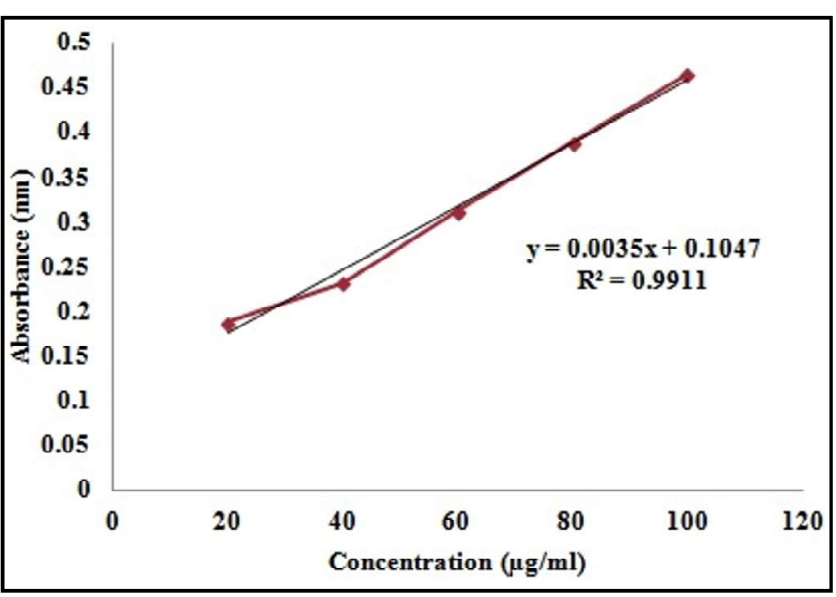

Figure 10: Caliberation of Rutin.

\section{Discussion}

Standardization of a crude drug is an important parameter to establish authenticity, with aspect to purity and quality. It includes various parameters such as botanical, physical, chemical, and biological. Among all these parameters, organoleptic and histochemical evaluations considered as an integral part and preliminary approach to check the correct identification of herbal drugs.

C. carandas (Apocynaceae) is extensively used in traditional herbal medicine for the treatment of various diseases. Such studies of pharmacopeial standards are found to be helpful for the proper identification and even authentication of the fruit of the same plant. The schematic diagram has been described in the Figure 11. The ray diagram and transverse section of $C$. carandas fruits with different reagents were performed and studied for the first time in an informative manner, using a phase-contrast microscope. The macroscopical characters revealed the color, taste, texture, and type of fruit. Microscopical studies indicated the presence of the cuticle layer, epidermis, parenchyma cells, and mesocarp cells, respectively. The presence of flavidovascular bundles, sclereids, and calcium oxalate crystals are the characteristic features of fruit powder. Physical parameters, including ash value, moisture content, foaming index, foreign organic matter, and extractive values performed in this research work. Such parameters used to detect the quality and purity of the crude drug, which correlated with their adulteration and substitution either with the harmful substances or allied drugs. Ash value can define as the residue which remains after the incineration of crude drugs. Therefore, it provides any impurities, including inorganic components or even earthy matter.

Foreign matter is the method of evaluating appreciable quantities of potent foreign matter, animal excreta, other than plant parts in the sample. 
Moisture content means the presence of an excess amount of water in the sample, which may lead to the activation of enzymes, which ultimately enhances the proliferation of living organisms. The presence of microorganisms may cause rapid deterioration of the sample. The moisture content of the dried fruit powder was found to be $9.69 \% \mathrm{w} / \mathrm{w}$.

The fluorescence study reveals various characteristic fluorescence observation under visible light and UV light (short and long wavelength), which found helpful in the identification of adulteration of the plant. The fluorescent analysis observed under visible light and UV light using different chemical reagent(s) showed various colors. Results obtained from the fluorescent analysis of fruit powder showed pink color in daylight whereas yellow and brown color in short and long wavelength.

The preliminary phytochemical evaluation was performed to identify the presence of noted chemical constituents in the literature in the fruits of the plant. It shows the presence of alkaloids, saponin glycosides, flavonoids, tannins, and phenolic compounds, fats, and oils, volatile oils, carbohydrates, gums, mucilage, and reducing sugars.

Generally, plants with flavonoids as well as phenols are with highly effective radical scavenging and antiradical properties (Kähkönen et al., 1999; Rice-Evans et al., 1995; Zübeyir Huyut et al., 2017). Investigations revealed that the phenolic components, recognized as antiradical agents may possess several biological activity (RiceEvans et al., 1996). The presence of such secondary metabolites in fruit herbs may reflect their significant finding of the present work. The main objective of this study is to provide a pharmacognostic evaluation with the determination of TPC and TFC in C. carandas fruit extracts. The limitation of this study is that TLC and HPLC have not performed along with the application of standard chemical constituents (markers). From the phytochemical evaluation, it was noted the presence of various classes of chemical components present in extracts. Therefore, by the application of phytochemical markers, different chemical constituents will be able to identify and even studied in the future.

\section{Conclusion}

Through, the present research, the morphology and anatomy of $C$. carandas fruit have been evaluated, which gives an essential diagnostic way to identify the purity and quality of the crude drug. It also imparts some information for the evaluation of adulterants and even substituent of allied drugs. The analysis of phenolic and flavonoid contents in the fruit extract of the plant may reveal their significant importance as an antiradical agents. However, the isolation of various bioactive secondary metabolites would assist to ascertain its potency and safety as a lead candidate of antioxidant property for pharmaceutical uses.

\section{Acknowledgements}

The authors are very much thankful to GLA University, Mathura281406 , for providing wonderful facilities to do research works.

\section{Conflict of interest}

The authors declare that there are no conflicts of interest in the course of conducting the research. All the authors had final decision regarding the manuscript and decision to submit the findings for publication.

\section{References}

Agarwal, T.; Singh, R.; Shukla, A.D. and Waris, I. (2012). In vitro study of antibacterial activity of Carissa carandas leaf extracts. Asian J. Plant Sci. Res., 2:36-40.

Anupama, N.; Madhumitha, G. and Rajesh, K.S. (2014). Role of dried fruits of Carissa carandas as anti-inflammatory agents and the analysis of phytochemical constituents by GC-MS. BioMed Research International, pp:2014. doi.org/10.1155/2014/512369.

Arif, M.; Kamal, M.; Jawaid, T.; Khalid, M.; Singh, S.K. and Kumar, A. (2016). Carissa carandas Linn. (Karonda): An exotic minor plant fruit with immense value in the nutraceutical and pharmaceutical industries. Asian J. Biomed. Pharm. Sci., 6(58):14-19.

Atanassova, M.; Georgieva, S. and Ivancheva, K. (2011). Total phenolic and total flavonoid contents, antioxidant capacity and biological contaminants in medicinal herbsm. J. Chem. Technol. Metall., 46(1):81-88.

Begum, S.; Syed, S.A.; Siddiqui, B.S.; Sattar, S.A. and Choudhary, M.I. (2013). Carandinol: First isohopane triterpene from the leaves of Carissa carandas L. and its cytotoxicity against cancer cell lines. Phytochemistry Letters, 6:91-95.

Chanchal, K.M.; Shrivastava, B. and Sasmal, D. (2013). Pharmacognostical standarization and phytochemical identification of fruit and root of Carissa carandas Linn. Int. J. Pharm. Pharm. Sci., 5:347-350.

Chatterjee, A. and Satyesh, C.P. (1994). The treatise on Indian medicinal plants. National Institute of Science Communication and Information Resources, New Delhi, pp:123-134.

Cook, N.C. and Samman, S. (1996). Flavonoids-chemistry, metabolism, cardioprotective effects, and dietary sources. J. Nutr. Biochem., 7:66-76.

Edeoga, H.O.; Okwu, D.E. and Mbaebie, B.O. (2005). Phytochemical constituents of some Nigerian medicinal plants Afric. J. Biotech., 4(7):685-688

Elizabeth, A.A. and Kelly, M.G. (2007). Estimation of total phenolic content and other oxidation substrates in plant tissues using Folin-Ciocalteu reagent. Nat. Protoc., 2(4):875-877.

Evan, W.C. (2006). Trease and evans pharmacognosy. Elsevier Publication, New Delhi, pp:112-117.

Hati, M.; Jena, B.K.; Kar, S. and Nayak, A.K. (2014). Evaluation of antiinflammatory and anti-pyretic activity of Carissa carandas L. leaf extract in rats. Journal of Pharmaceutical, Chemical and Biological Sciences, 1:18-25.

Iygengar, M.A. and Nayak, S.C. (1997). Anatomy of crude drugs. B.S. Publications, Hyderabad, pp:11-17.

Kähkönen, M.P.; Hopia, A.I.; Vuorela, H.J.; Rauha, J.; Pihlaja, K.; Kujala, T.S. and Heinonen, M. (1999). Antioxidant activity of plant extracts containing phenolic compounds. J. Agric. Food Chem., 47(10): 3954-3962.

Khandelwal, K.R. (2012). Practical pharmacognosy-techniques and experiments. Nirali Prakashan Pune, 25:1-9. 
Kokate, C. K. (1999). Pharmacognosy. Nirali Prakashan Pune, pp:97-101.

Kokate, C. K. (2005). Practical pharmacognosy. Vallabh Prakashan, New Delhi, pp:32-38.

Kumar, R.S.; Sivakuma, T.; Sunderem, R.S.; Gupta, M.; Murugesh, K.; Rajeshwa, Y.; Kumar, M.S. and Kumar, K.A. (2005). Antioxidant and antimicrobial activities of Bauhinia racemosa L. stem bark. Braz. J. Med. Biol. Res., 38:1015-1024.

Ling-jia, Z.; Wei, L.; Su-hui, X.; Wei, Liu.; Su-hui, Xiong.; Jie, T.; Zhao-huan, L.; Ming-xia, X.; Bo-hou, X.; Li-mei, L. and Duan-fang, L. (2018). Determination of total flavonoids contents and antioxidant activity of Ginkgo biloba leaf by near-infrared reflectance method. Int. J. Anal. Chem., pp:8195784.

Mimica-Dukic, N.; Bozin, B.; Sokovic, M. and Simin, N. (2004). Antimicrobial and antioxidant activities of Melissa officinalis L. (Lamiaceae) essential oil. J. Agric. Food Chem., 52(9):2485-2489.

Modak, M.; Dixit, P.; Paul, T. and Devasagayam. (2007). Indian herbs and herbal drugs used for the treatment of diabetes. J. Clin. Biochem. Nutr., 40:163-173.

Omale, J. and Okafor, P.N. (2008). Comparative antioxidant capacity, membrane stabilization, polyphenol composition and cytotoxicity of the leaf and stem of Cissus multistriata. Afric. J. Biotech., 7(17):3129-3133.

Pandey, M.M.; Rastogi, S. and Rawat, A.K.S. (2013). Indian traditional ayurvedic system of medicine and nutritional supplementation. Evid. Based Complementary Altern. Med., pp:2013 doi:10.1155/ $2013 / 376327$.

Rangari, V.D. (2000). Pharmacognosy and phytochemistry. Carrier Publication, New Delhi, pp:55-67.
Rice-Evans, C.A.; Miller, N.J. and Paganga, G. (1996). Structure-antioxidant activity relationships of flavonoids and phenolic acids. Free Radic. Biol. Med., 20(7):933-956.

Rice-Evans, C.A.; Miller, N.J.; Bolwell, P.J.; Bramley, P.M. and Pridham, J.B. (1995). The relative antioxidant activities of plant-derived polyphenolic flavonoids. Free Radic. Res., 22(4):375-383.

Sadek, Y.B.; Choudhury, N. and Shahriar, M. (2013). Biological investigations of the leaf extracts of Carissa carandas. ITPRT, 5:97-105.

Saha, R.; Hossain, L.; Bose, U. and Rahman, A.A. (2011). Neuropharmacological and diuretic activities of Carissa carandas L. leaf. Pharmacologyonline, 2:320-327.

Singh, A. and Uppal, G.K. (2015). A review on Carissa carandasphytochemistry, ethnopharmacology, and micropropagation as conservation strategy. Asian J Pharm Clin Res., 8(3):26-30.

Tesfaye, T. and Ravichandran, Y.D. (2018). Traditional uses, pharmacological action, and phytochemical analysis of Carissa carandas Linn.: A review. Nat. Prod. Chem. Res., 6(5). doi: 10.4172/2329-683 6.1000334 .

Virmani, R.; Virmani, T.; Singh, C.; Sorout, G. and Gupta, J. (2017). Hidden potential of natural herb Carissa carandas (Karonda). Research in Pharmacy and Health Sciences, 3:294-302.

WHO (1992). Quality control methods for medicinal plant material. Geneva: WHO, pp:22-34.

Zübeyir Huyut, pükrü Beydemir, and Ýlhami Gülçin. (2017). Antioxidant and antiradical properties of selected flavonoids and phenolic compounds. Biochem. Res. Int., pp:2017. Article ID 7616791.

Citation Sonia Singh, Meenakshi Bajpai and Pradeep Mishra (2020). Pharmacognostical evaluation of indigenous herbal drug: Carissa carandas Linn. fruit . Ann. Phytomed., 9( 2):263-270. http://dx.doi.org/10.21276/ap.2020.9.2.25 